\title{
Artificially accumulated B-catenin inhibits proliferation and induces neurite extension of neuroblastoma cell line NB-1 via up-regulation of trkA
}

\author{
SURASAK SANGKHATHAT ${ }^{1,3}$, KEIGO NARA $^{1}$, TAKESHI KUSAFUKA ${ }^{2}$, \\ AKIHIRO YONEDA $^{1}$ and MASAHIRO FUKUZAWA ${ }^{1}$ \\ ${ }^{1}$ Department of Pediatric Surgery, Osaka University Graduate School of Medicine, 2-2 Yamadaoka, Suita, Osaka 565-0871; \\ ${ }^{2}$ Division of Pediatric Surgery, Department of Surgery, Nihon University School of Medicine, 30-1 Oyaguchikami-machi, \\ Itabashi-ku, Tokyo 173-8610, Japan; ${ }^{3}$ Pediatric Surgery Unit, Department of Surgery, Faculty of Medicine, \\ Prince of Songkla University, Hadyai, Songkhla, Thailand
}

Received May 2, 2006; Accepted July 25, 2006

\begin{abstract}
Recent evidence suggests an association between up-regulation of $\beta$-catenin/Wnt signaling pathway and neuronal differentiation of neuroblastoma. We overexpressed $B$ catenin into a human neuroblastoma cell line NB-1 and observed its effect on cellular morphology, growth potential and alteration in a known differentiation related gene, trkA. Expression plasmids containing wild-type and mutated forms of B-catenin gene were transfected into NB-1 cells, using liposome-based transfection method. The mutated forms were a deletion of three nucleotides of codon 45 and a large deletion involving the whole exon 3 . In the transient transfection model, cell viability assay demonstrated significant negative effect of mutated $\beta$-catenin transfection, but not wild-type, on the cell proliferation. To investigate impacts of B-catenin overexpression in detail, a stable transfection model was established. Clones with comparable expression of $\beta$-catenin at the mRNA level were selected. Only the selected clones with mutated form of $B$-catenin exhibited neurite extension pattern and stunned cell proliferation, in association with higher accumulation of total cellular $\beta$ catenin protein as evidenced by Western blot and immunocytochemistry. Cell cycle progression demonstrated significantly higher G0-G1 fraction in each stable cell clone with $\beta$-catenin expression plasmid. In addition, retarded G1/S transition was observed exclusively in the cell clones with mutated form. Concomitantly with overexpressed B-catenin,
\end{abstract}

Correspondence to: Professor Takeshi Kusafuka, Division of Pediatric Surgery, Department of Surgery, Nihon University School of Medicine, 30-1 Oyaguchikami-machi, Itabashi-ku, Tokyo 173-8610, Japan

E-mail: kusafuka@med.nihon-u.ac.jp

Key words: neuroblastoma, ß-catenin, tyrosine kinase, neuronal differentiation up-regulations of trkA and Ha-ras were also identified. Our study suggests a potential availability of $\beta$-catenin/Wnt signaling pathway as a target of molecular manipulation for treatment of high-risk neuroblastoma and a potential association between the pathway and the trkA/neurotrophin cascades.

\section{Introduction}

Neuroblastoma is one of human cancers with high diversity of disease progression which is associated with its heterogeneity in the biology (1). Differentiation of malignant neuroblastic cells can be found varying from poorly differentiated neuroblasts to mature ganglion cells. Moreover, inverse relationship between the aggressiveness of a neuroblastoma and its maturation has been well defined in in vitro experiments as well as in clinical series $(2,3)$. Undifferentiated neuroblastoma tends to metastasize and responds poorly to chemotherapeutic agents while tumors containing more mature cells are less aggressive. In addition, some neuroblastomas have a potency to regress spontaneously from an undifferentiated state to a completely benign cellular appearance $(4,5)$. Manipulating the level of differentiation in this kind of malignancy is, therefore, one of the molecular therapeutic strategies. Although various extrinsic factors has been demonstrated to induce neuronal differentiation such as transretinoic acid ( $t \mathrm{RA}$ ), lithium, and various growth factors (6-8); intrinsic signaling pathways which govern the differentiation process in a neuroblastoma remains less well elucidated.

Roles of tyrosine kinase signaling in growth and differentiation of neuroblastoma have been considerably explored. In this pathway, nerve growth factor (NGF) receptor, tyrosine kinase-A (trkA), is the main player that binds to NGF to activates downstream transcription of genes related to survival or differentiation $(1,9)$. In addition, up-regulation of trkA was also demonstrated in the model of $t$ RA induced neuronal differentiation (10).

Wnt-signaling pathway plays an important role in neuronal development process in vertebrates. In the canonical Wnt/ßcatenin cascade, $\beta$-catenin acts as a central molecule that activates transcription of downstream growth-promoting genes. 
Intracellular level of $\beta$-catenin is tightly regulated by its phosphorylation through casein kinase and glycogen synthase kinase-3ß (GSK-3ß) (11). ß-catenin is expressed constitutively in neurons and neuroblastoma (12). Lines of evidence suggested that up-regulation of Wnt-signaling pathway has a potential effect on differentiation status of neuroblasts. In mouse neuroblastoma cells treated by lithium chloride, inhibition of GSK-3ß by the chemical compound leads to an accumulation of $B$ catenin and morphological differentiation (7). A recent study demonstrated that $B$-catenin plays a role in promoting neuronal differentiation of murine neural stem cells (13). Taken together we hypothesized that it should be possible to induce even human neuroblastoma cells into a differentiation process by manipulating ß-catenin itself. In this study, we transfected expression plasmids containing full-length wild-type and mutated B-catenin into a neuroblastoma cell line NB-1 and found negative impact of overexpression of $\beta$-catenin on cell progression in transient transfection experiments. In stably transfected cell lines, multidirectional neurite extension, together with significant decrease in cellular proliferation rate and retarded cell-cycle progression were observed. Moreover, alteration in the expression of trkA protein and one of its downstream members, Ha-ras, in association with B-catenin expression suggested a potential link between Wnt signaling and neurotrophin signaling cascades.

\section{Materials and methods}

Plasmid construction. Plasmid pEGFP-C1-BCAT-wt and pEGFP-C1-BCAT-del Exon3 were constructed by cloning cDNA sequence of wild-type $B$-catenin and $B$-catenin with a large deletion on its exon 3 , respectively, into a vector pEGFP-C1 (BD Biosciences Clontech, Palo Alto, CA, USA). The cDNA sequences were derived from digestion of plasmids pUHD10-3-BCAT-wt, and pUHD10-3-BCAT-del Exon3 [kindly provided by Dr Yataka Nagasawa, Biomedical Research Center, Osaka University, Japan (14)] with restriction enzymes SacII and BamHI. Subcloning of the B-catenin sequences into pEGFP-C1 was done following digestion of the vector with the same enzymes. To construct pEGFP-C1BCAT-delTCT45, a plasmid with a deletion mutation at codon 45 on exon 3 of $ß$-catenin sequence, pEGFP-C1BCAT-wt was used as a template for the mutagenesis experiment, using QuickChange ${ }^{\mathrm{TM}}$ site-directed mutagenesis kit (Stratagene, Cedar Creek, TX, USA), following the manufacturer's instructions. Blank vector pEGFP-C1 was employed as a mock control.

Cell culture. Human neuroblastoma cells, NB 1 (Riken Cell Bank, Ibaraki, Japan), were cultured in Dubecco's modified Eagle's medium (DMEM, Nacali Tesque, Kyoto, Japan) supplemented with $10 \%$ fetal bovine serum (FBS, Cellect, MP Biomedical, Inc., Eschwege, Germany) and $100 \mathrm{U} / \mathrm{ml}$ penicillin, $100 \mu \mathrm{g} / \mathrm{ml}$ streptomycin (Nacali Tesque), in humidified $5 \% \mathrm{CO}_{2}$ at $37^{\circ} \mathrm{C}$. The cells were fed every 3 days and subcultured at $80 \%$ confluence.

\section{Transient transfection experiments}

WST-1 assay of cell viability after transient transfection with $\beta$-catenin plasmid. To study effects of various types of $\beta$-catenin overexpression in NB1 cells, transient transfection experiment was done. Briefly, cells were seeded on a 96-well plate at the density of $10^{4}$ cells/well. Simultaneously with seeding, transfection of each plasmid was done by using TransIT-Neural reagent (Mirus Corp., Madison, WI, USA) at the concentration of $0.15 \mu \mathrm{g}$ of plasmid/10 $\mu \mathrm{l}$ of transfection reagent/well. Twenty-four hours later, a follow-on transfection was repeated with the same mixture. With this transfection protocol, the efficiency was approximately $30-40 \%$ as evaluated by a transfection of blank pEGFP vector (data not shown). To determine cell progression ability in each transfection group, WST-1 viability assay (Nacali Tesque) was performed at day 3,5 and 7 post-transfection. After $10 \mu \mathrm{l}$ of WST- 1 solution was added to each well, the plate was incubated at $37^{\circ} \mathrm{C}$. The absorbency of the treated samples against blank controls was measured with an immunoreader (Immuno Mini NJ-2300, Nippon Inter-Med K.K., Tokyo, Japan) with $414 \mathrm{~nm}$ as a detection wavelength and $630 \mathrm{~nm}$ as a reference wavelength.

Gene expression assays by real-time reverse transcriptionpolymerase chain reaction ( $R T-P C R)$. Total cellular RNA was prepared by RNAqueous RNA isolation kit (Ambion, Austin, TX, USA), according to the manufacturer's protocol. First-strand cDNA was synthesized from $1 \mu \mathrm{g}$ of total-RNA using MMLV Reverse Transcriptase (Clontech Laboratories, Palo Alto, CA, USA) and oligo(dT) primers. Twenty-four hours prior to transfection, $5 \times 10^{5}$ cells of NB-1 were seeded in each well of a 6-well plate. Transfection was performed in the same protocol as for the transient transfection model described above. After $48 \mathrm{~h}$, cells were harvested for RNA extraction and cDNA synthesis. To quantitate the level of mRNA of B-catenin, real-time RT-PCR was performed on an ABI PRISM 7700 Sequence Detection System together with Sequence Detector V1.7 software (PE Applied Biosystems, Inc., San Jose, CA, USA). Primers and TaqMan probes used in this study were presented previously (15). Human GAPDH was used as an internal control. The mRNA copy numbers of B-catenin were standardized by the copy number of GAPDH in each sample.

\section{Stable transfection model}

Selection of stably transfected cell lines. Cells were seeded at $1.5 \times 10^{5}$ cells/well in 6-well plate. Each plasmid $(3 \mu \mathrm{g})$ was incubated with $6 \mu 1$ of TransIT-Neural reagent in $200 \mu 1$ of serum-free media for $15 \mathrm{~min}$ before adding to each well. Approximately $24 \mathrm{~h}$ after the transfection, expression of green fluorescent protein was checked under an ultraviolet microscope and the culture media were then replaced by selection media containing $750 \mu \mathrm{g} / \mathrm{ml}$ of G418 (geneticin, Nacali Tesque). Cells were maintained in the selection media for at least 14 days. The isolated G418 resistant cell clones were then selected and amplified in the media with $500 \mu \mathrm{g} /$ $\mathrm{ml}$ of $\mathrm{G} 418$.

To confirm the existence of transfected $B$-catenin gene, DNA was extracted from each selected cell clone with High Pure PCR template preparation kit (Roche Inc., Indianapolis, IN, USA), following the supplier's instructions. PCR was performed using the primer set BCAT3/4 $(16,17)$ which were placed on exon 2 and 4, flanking the whole length of exon 3. In a cell colony with extrinsically transfected $B$-catenin 
sequence, an additional smaller PCR product band of cDNA size (either wild-type or truncated size) appears whereas in a parental cell line and the cell colonies stably transfected with blank GFP vector, PCR gave only a single large band amplified from genomic DNA (data not shown). The smaller band from each sample was cut and the genotype confirmed by direct sequencing. Cell clones with comparable expression level of B-catenin at the mRNA level as verified by real-time RT-PCR were selected from each group. Selected stable cell clones with empty pEGFP-C1, pEGFP-C1-BCAT-wt, pEGFP-C1-BCAT-delTCT45 and pEGFP-C1-BCAT-del Exon3 were designated as mock, wild-type, del-TCT45 and del-Exon3, respectively.

Immunocytochemistry and cytochemistry. Stably transfected cells in each group were plated on a 4-chamber glass slide (BD Falcon, Becton-Dickinson \& Co., San Jose, CA, USA) at the density of $10^{5}$ cells/chamber. Four days after plating, cells were fixed in $4 \%$ paraformaldehyde and slides were rinsed in PBS, permeabilized with $1 \%$ Triton X-100 for $1 \mathrm{~h}$, then blocked with $10 \%$ normal rabbit serum for $30 \mathrm{~min}$ before incubation with anti- $\beta$-catenin antibody $(0.5 \mu \mathrm{g} / \mathrm{ml}$; Transduction Laboratories, Lexington, KY, USA) overnight at $4^{\circ} \mathrm{C}$. For visualization, FITC-conjugated rabbit anti-mouse immunoglobulins (6 mg/ml; Dako, Glostrup, Denmark) were used as second antibody. To study the change in cell morphology, slides were incubated in PBS containing $0.002 \%$ of Phalloidin-Tetramethylrhodamine-B-isothiocyanate (TRTC) (Sigma-Aldrich Corp., St. Louis, MO, USA) in order to stain filamentous actin, followed by $0.02 \%$ diamidino-2phenylindole (Nacali Tesque) for nuclear staining, then washed and mounted with fluorescence mounting medium (DakoCytomation, Tokyo, Japan). Photography was taken with Keyence VB6000 digital photography system (Keyence, Tokyo, Japan) attached to Nikon Eclipse C1000 microscope (Nikon, Tokyo, Japan). Negative control neglected the Bcatenin antibody, but followed the other staining procedures.

Study of $\beta$-catenin protein alteration and simultaneous change of trkA and Ha-ras expression by Western blotting. Total cellular extracts from each stably transfected line were collected using RIPA lysis buffer (Upstate, Lake Placid, NY) supplemented with protease inhibitor cocktails. Insoluble material was removed by centrifugation at 14,000 x $\mathrm{g}$ for $10 \mathrm{~min}$, and the supernatant was used for Western blot analysis. Protein concentration was determined with the BCA protein assay set (Pierce, Rockford, IL, USA). Approximately $20 \mu \mathrm{g}$ of total protein were loaded into each lane on a $10 \%$ sodium dodecyl sulfate-polyacrylamide gel electrophoresis and subsequently transferred onto a polyvinyl difluoride membrane. The membrane was incubated with primary antibody for $1 \mathrm{~h}$ at room temperature. Primary antibodies used in this study were anti- $\beta$-catenin monoclonal antibody (Transduction Laboratories) at a dilution of $0.5 \mu \mathrm{g} / \mathrm{ml}$, trkA (H-190) polyclonal antibody $(0.2 \mu \mathrm{g} / \mathrm{ml}$, Santa Cruz Biotechnology, Inc. Santa Cruz, CA, USA), and Ha-ras (C20) polyclonal antibody $(0.2 \mu \mathrm{g} / \mathrm{ml}$, Santa Cruz Biotechnology, Inc.). The membrane was then washed three times and incubated with the horseradish peroxidase conjugated antibody of appropriate species (Dako) for $1 \mathrm{~h}$. After three washes with TBS, the signals were developed using an enhanced chemiluminescence system (ECL plus, Amersham Biosciences, Inc., Piscataway, NJ, USA) and visualized after exposure to Hyperfilm (Amersham Biosciences, Inc.).

Growth study by trypan blue exclusion technique. To study the growth characteristic of each stable cell clone, $5 \times 10^{4}$ cells from each clone group were plated in triplicate in $1 \mathrm{ml}$ of complete media in 24-well plates. Cells were trypsinized and then counted every $24 \mathrm{~h}$ for 10 days using a hemocytometer and trypan blue exclusion technique.

Cell cycle analysis. Each stably transfected NB-1 clone $\left(3 \times 10^{5}\right.$ cells/well) was seeded in a 6-well plate. At 24, 48 and $72 \mathrm{~h}$, the cells were washed twice with ice-cold PBS, harvested, fixed with ice-cold PBS in $70 \%$ ethanol, and stored at $4^{\circ} \mathrm{C}$. For a flow cytometric analysis, the cells were incubated with $0.1 \mathrm{mg} / \mathrm{ml} \mathrm{RNase-A}$ at $37^{\circ} \mathrm{C}$ for $30 \mathrm{~min}$, stained with $50 \mu \mathrm{g} / \mathrm{ml}$ propidium iodide for $30 \mathrm{~min}$ on ice, and then measured using a FACScan flow cytometer (BectonDickinson, Franklin Lakes, NJ, USA) with CellQuest software. Analysis of cell cycle was assisted with FlowJo v.4.4.4 software (Tree Star Inc., Ashland, OR, USA). The experiments were performed three times and the average values are presented.

Apoptosis evaluation. For stable cell clones, apoptosis assay was performed semi-quantitatively by using ApopTag Plus Fluorescein In Situ apoptosis detection kit (Serologicals Corp., Norcross, GA, USA), according to the manufacturer's protocol which is based on TUNEL principle. Briefly, cells were seeded in equal amount in four-chamber glass slides. After 3 days, slides were fixed with paraformaldehyde and subsequently reacted with dioxygenin-labeled terminal deoxynucleotidyl transferase, followed by fluorescein conjugated anti-dioxygenin antibody binding. Positive cells harboring apoptotic bodies were counted under a fluorescence microscope.

Evaluation of reversibility of $\beta$-catenin effect on proliferation by $\beta$-catenin siRNA transfection. Del-TCT45 cells were plated at the density of $10^{4}$ cells/well in a 96-well plate. B-catenin siRNA oligonucleotides (SMARTpool, Dharmacon, Lafayatte, CO, USA) were transfected using HiPerFect transfection reagent (Qiagen, Inc., Valencia, CA, USA) at the final concentration of $100 \mathrm{nM}$ of siRNA in serum-free DMEM. Details regarding sequences of siRNA can be found in our previous report (15). Sham transfection using HiPerFect alone and transfection of negative control siRNA (B-Bridge International, Inc., Sunnyvale, CA, USA), which has no significant homology to any known sequences in human genome, were also performed. On the third day of treatment, comparative cell progression was evaluated by WST-1 viability assay.

To evaluate knock-down efficiency at the mRNA level, $5 \times 10$ cells were seeded on each well of a 6 -well plate. siRNA treatment including controls was applied in the same protocol as above. Cells were harvested at $48 \mathrm{~h}$ after the treatment for RNA extraction and real-time RT-PCR.

Data analysis and statistics. Unless stated otherwise, all experiments were performed in triplicate under similar 
A

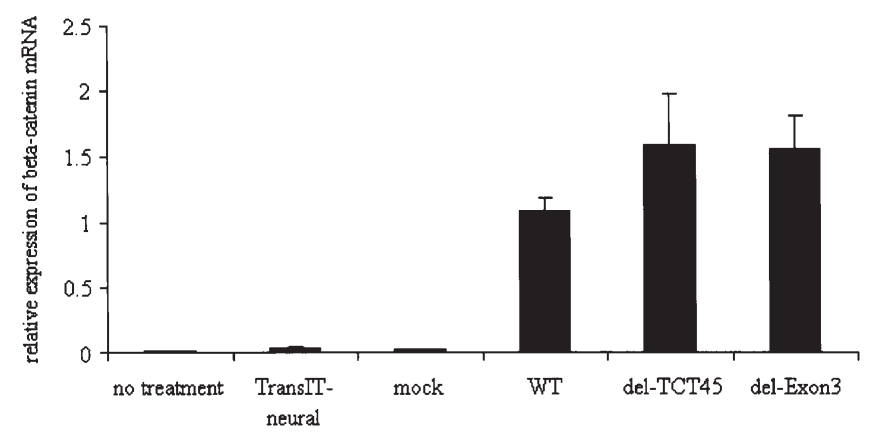

B

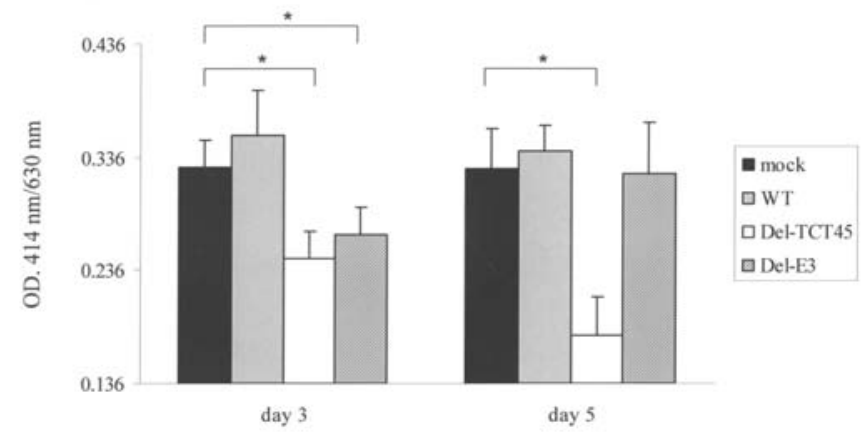

Figure 1. (A), Results of real-time RT-PCR demonstrating mRNA expression of $\beta$-catenin relative to GADPH in NB-1 cells increased in comparable levels in all three groups transfected with $\beta$-catenin expression plasmid. (B), WST-1 assay demonstrated significant lower amount of viable cells in those groups transfected with point-mutated and large truncated B-catenin (delTCT45 and del-Exon3 plasmids) ( ${ }^{*}$ p-value $\left.<0.01\right)$.

conditions. Data are presented as means together with standard deviation for each parameter. The statistical analysis was performed by the unpaired Student's t-test, and statistical significance was considered when the p-value was $<0.05$ or 0.01 , as appropriate.

\section{Results}

Overexpression of $\beta$-catenin suppressed proliferation of $N B-1$ cells. After transfection, mRNA expression of $\beta$-catenin in NB-1 cells increased in comparable levels in all three groups transfected with B-catenin expression plasmid (Fig. 1A). Synchronous with this overexpression, WST-1 assay demonstrated significant lower amount of viable cells in those groups transfected with point-mutated and large truncated B-catenin (del-TCT45 and del-Exon3) (Fig. 1B). It was noted that the group transfected with point-mutated plasmid exhibited stronger and longer growth inhibition effect than the plasmid with whole exon truncation.

Accumulation of $\beta$-catenin protein in stable clones with mutated $\beta$-catenin plasmid. In the stable transfection model, cell clones with comparable expression of $\beta$-catenin were selected from each group. Real-time RT-PCR revealed slightly lower mean expression level of $B$-catenin in cell groups that were transfected with del-TCT45 and del-Exon3 plasmids (Fig. 2A). Western blot study showed higher amount

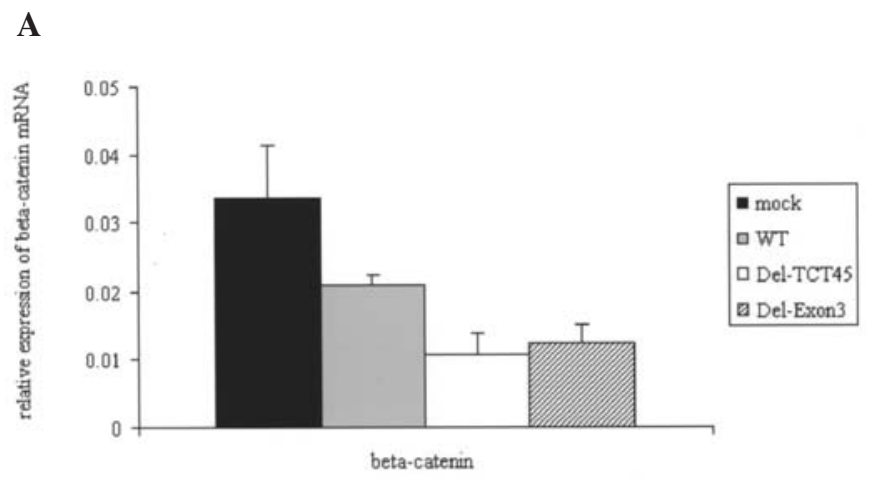

B
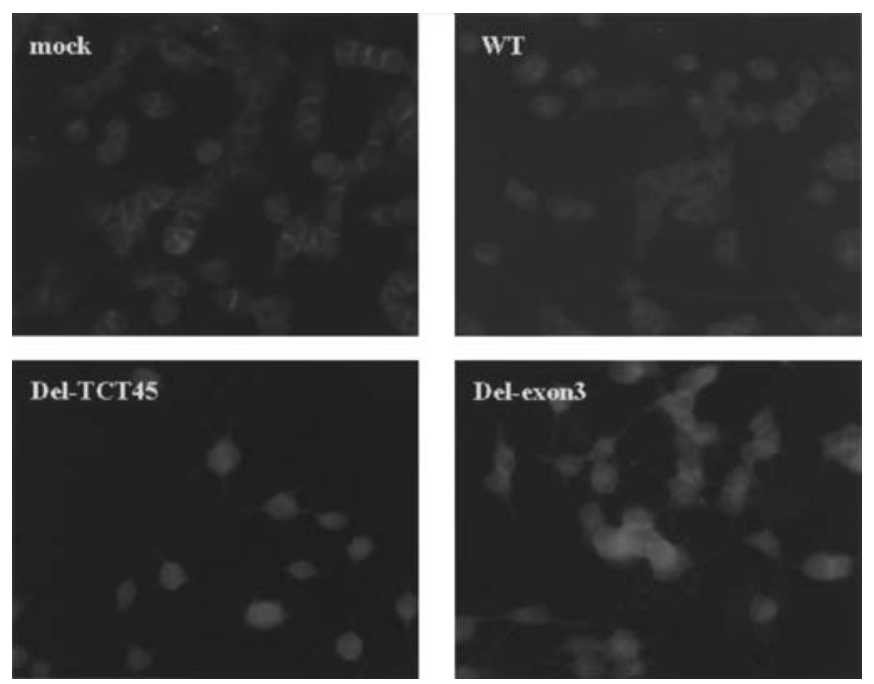

Figure 2. (A), Average relative expression of stable clones in each transfection group which showed slightly lower mean expression level in cell groups that were transfected with del-TCT45 and del-Exon3 plasmids. (B), Conversely, B-catenin immunoreactivity in the del-TCT45 and the del-Exon3 was paradoxically more intense than the mock and the wildtype groups. Note that the localization of $\beta$-catenin in mock control was mostly membrane localization whereas in the del-TCT45 and the del-Exon3, accumulation appeared at the cytoplasm and the cell nucleus.

of $\beta$-catenin protein in the del-TCT45 and del-Exon3 clones (Fig. 3), which was consistent with immunocytochemistry results that demon-strated more intense immunoreactivity in those cell clones (Fig. 2B).

Growth retardation in NB-1 cells harboring artificial $\beta$-catenin expression was associated with an inhibition at G1/S phase transition. The growth study confirmed growth retardation in clones harboring mutated catenin (Fig. 6). At 48-h of seeding, the flow cytometry revealed a higher average G0-G1 phase fraction in all three clones transfected with $\beta$-catenin expression plasmid (Fig. 4A). In addition, a significant retardation of G1/S transition was observed in the two groups harboring mutated forms of $\beta$-catenin (Fig. 4B).

Multidirectional neurite extension in $\beta$-catenin transfected $N B-1$. Multidirectional neurite extension of NB-1 was observed in 2 groups of cell clone transfected with mutated $B$-catenin (del-TCT45 and del-Exon3) (Fig. 5). The same pattern of 


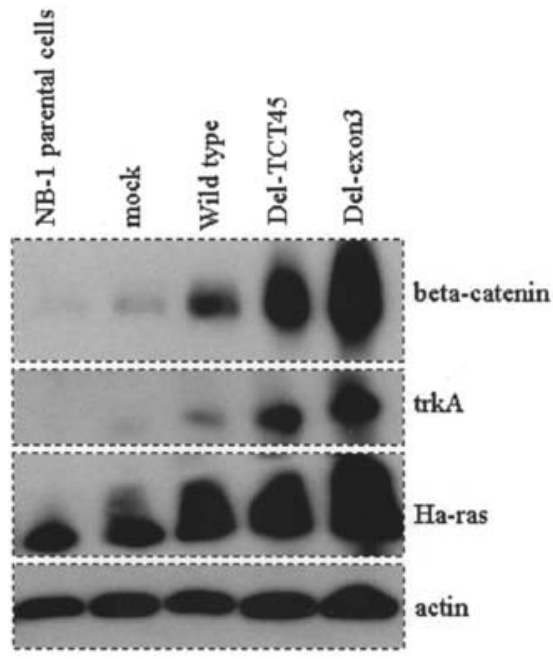

Figure 3. Western blot showed increased B-catenin protein in two groups of stable clone transfected with mutated $B$-catenin plasmid. Concomitantly with overexpression of $\mathrm{B}$-catenin protein, increased expressions of trkA and $\mathrm{Ha}-$ ras were identified.

A

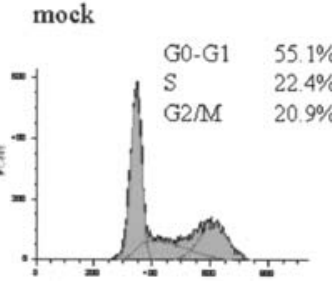

Del-TCT45
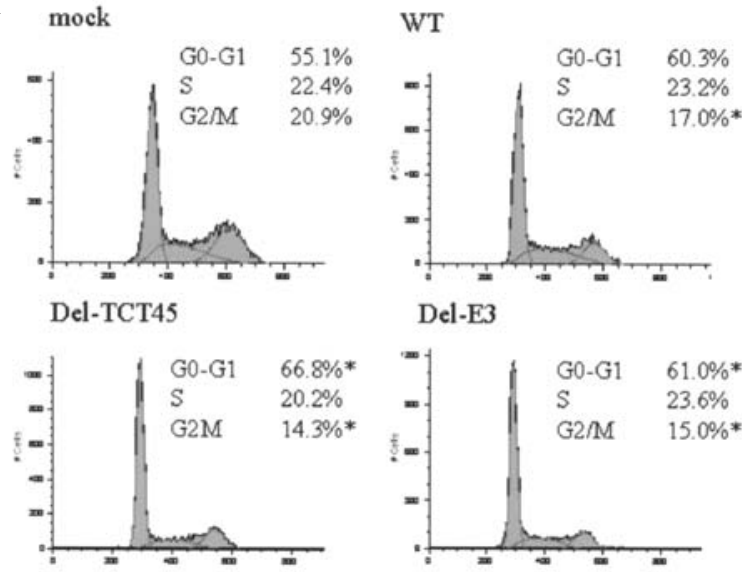

Del-E3

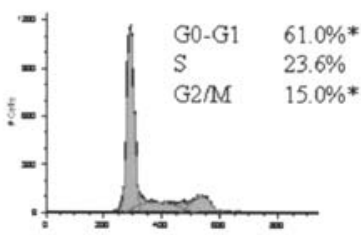

$\mathbf{B}$

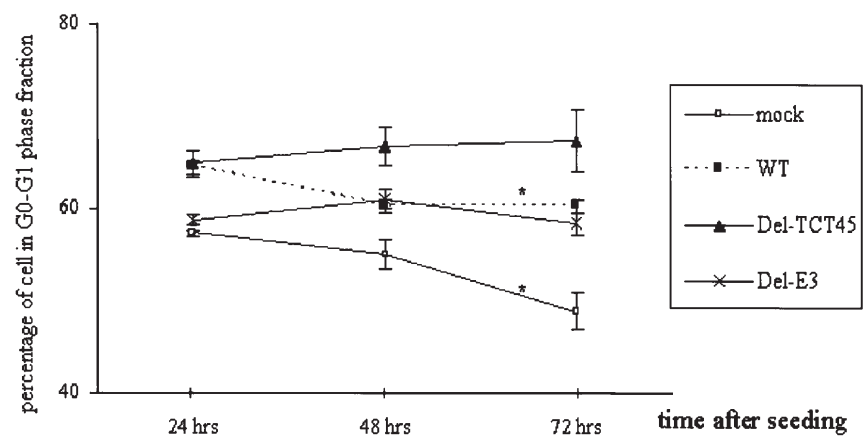

Figure 4. (A), Cell-cycle study by propidium iodide staining revealed significantly higher average G0-G1 phase fraction in all groups with $\beta$-catenin plasmid ("P-value $<0.05$ when compared to mock). (B), Time-course study showed retardation of G0-G1 decline in the del-TCT45 and the del-Exon3 groups. Average percentage of G0-G1 fraction at $72 \mathrm{~h}$ in mock and wildtype groups reduced significantly when compared with $24-\mathrm{h}\left({ }^{*} \mathrm{p}\right.$-value $\left.<0.05\right)$, however, there was no significant change of the values in the del-TCT45 and the del-Exon 3 groups.

morphologic change could be found in stable clones transfected with wild-type $B$-catenin plasmid, but to a lesser extent.
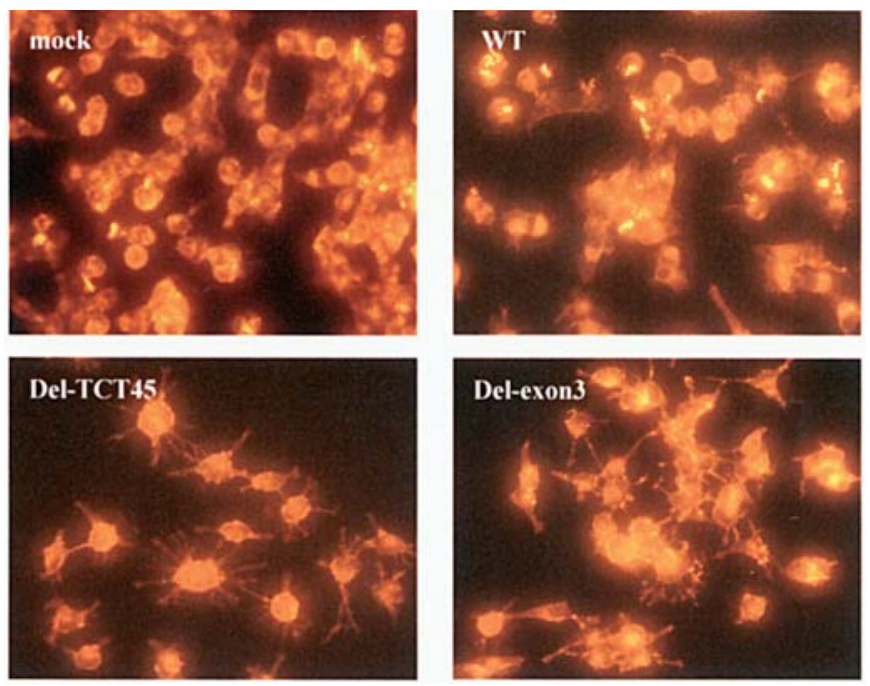

Figure 5. Phalloidin-TRTC staining of NB-1 exhibited multidirectional neurite extension of NB-1 was observed in two groups of cell clone transfected with mutated B-catenin (lower panel). The same pattern of morphologic change was found in stable clones transfected with wild-type B-catenin plasmid, but to a lesser extent.

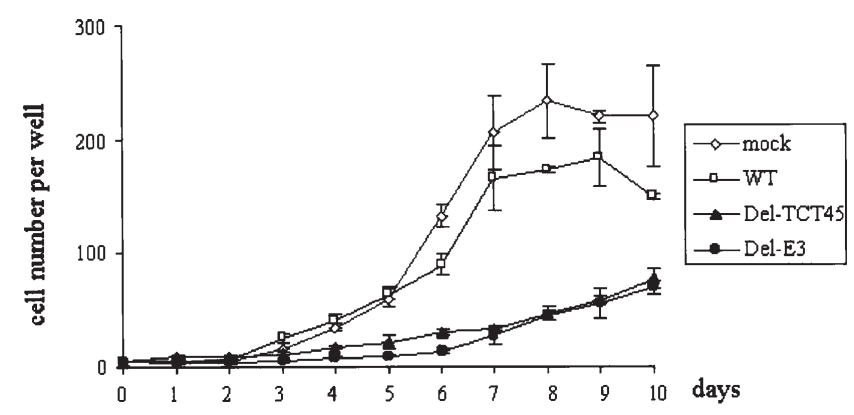

Figure 6. Time-course curve of growth comparison of stably transfected clones, using direct counting trypan blue exclusion. The study demonstrated significantly slower proliferation rate in the two groups with mutated ß-catenin.

Up-regulation of trkA and Ha-ras in cell clones with $\beta$-catenin accumulation. Western blot revealed increased expression of trkA and Ha-ras in all groups transfected with B-catenin expression plasmid (Fig. 3). The alteration of trkA was more obvious in clone groups transfected with mutated forms. Expression of Ha-ras was most intense in the clone transfected with large deletion form of ß-catenin.

Apoptosis was not increased in cell clones with $\beta$-catenin accumulation. The study found lower than three apoptotic bodies per high power field in each group of NB-1 cell clones and there was no difference among the groups.

Growth suppression in the del-TCT45 cell clone could be reversed by $\beta$-catenin siRNA. Real-time RT-PCR confirmed the efficiency of siRNA in post-transcriptional knock-down of B-catenin expression to $16 \%$ of the control level (Fig. 7A). This reduction resulted in significantly higher cell viability in the treatment group (Fig. 7B). 
A

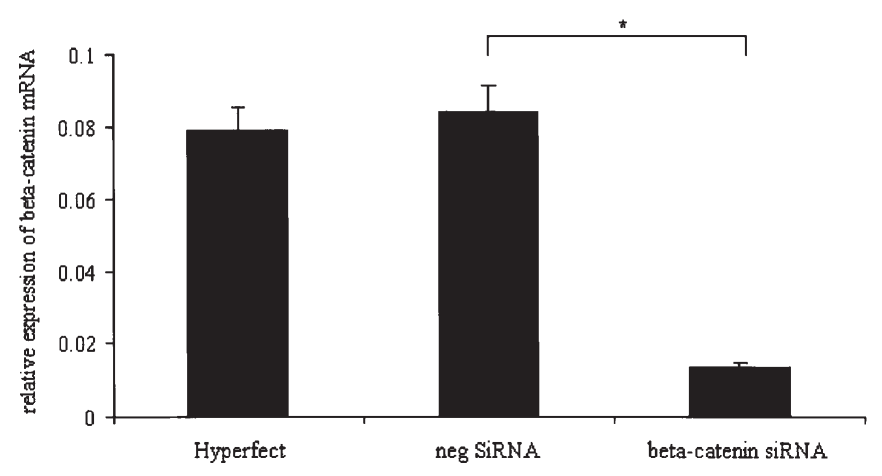

B

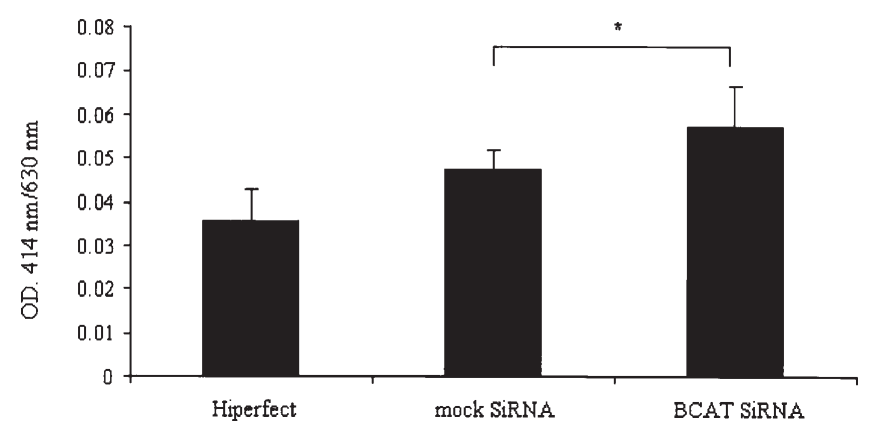

Figure 7. (A), A set of real-time RT-PCR results demonstrating reduction of B-catenin expression at the mRNA level when a clone of Del-TCT45 was transfected with $ß$-catenin siRNA. (B), Significantly positive change was seen in the treatment group as investigated by WST-1 ( ${ }^{*}$-value $\left.<0.05\right)$.

\section{Discussion}

Although neuroblastoma cells harbor uncontrolled proliferation and aggressive characteristic of malignancy, the behavior of developing neuron are retained in many aspects. Neuronal differentiation is one of the characteristics found to be varying from an individual tumor to another $(3,5)$. Correlation between level of differentiation and disease prognosis has been well addressed in clinical studies $(2,3)$.

Molecular mechanism regulating changes in cell morphology and growth in differentiation of neuroblastoma has not been thoroughly clarified, however, it is generally accepted that differentiation occur accompanied with an arrest of proliferation or escape from cell cycle (18). Certain extrinsic factors induce differentiation of neuroblastoma cells and one of the prototypes is $t$ RA, the mechanism of which is partly associated with increased expression of membranous receptor trkA. A previous study demonstrated that treatment with lithium chloride induced morphological differentiation of mouse neuroblastoma Neuro 2A cells (7). The study also found that neurite extension in lithium-treated cells was preceded by an accumulation of $\beta$-catenin via an inhibition of GSK-3ß and subsequent accumulation of $\beta$-catenin. In our study, a model of B-catenin accumulation was directly created in a human neuroblastoma cell line, NB-1, by forced expression of degradation-resistant forms of $\beta$-catenin.

We employed NB-1 in our model as this cell line is derived from human neuroblastoma with MYCN amplification and homozygous deletion of chromosome 1p (19), hence it represents tumors of poor prognosis. Moreover, this cell line is one of a few neuroblastoma cell lines that can be successfully transfected with liposome-based transfection reagent. In comparison with electroporation, less cytotoxicity caused by chemical transfection and considerable transfection efficiency makes the cell line more suitable for a direct study of the impact of extrinsic oligonucleotide induction. With normal culture conditions, the cells appeared as round to polygonal-shaped in monolayer. Neurite outgrowth is not usually found in early days after a subculture. However, we observed that a pattern of the outgrowth in nearly confluent condition was mostly bipolar extension of tough processes, which obviously differed from the multidirectional outgrowth of thin processes as found in our NB-1 lines transfected with B-catenin. To alleviate the factor of transfection efficiency, we studied alterations in morphology and cell kinetics using a stable transfection model. Western blot study found higher amount of B-catenin protein accumulation in the groups of cell clone transfected with mutated $\beta$-catenin, which was consistent with the immunostaining results. The phenomenon was concordant with the expectation that mutation involving the phosphorylation sites inhibits the degradation cascade and leads to stabilization and accumulation of $\beta$-catenin protein.

On transient transfection, although the transfection efficiency was limited, it was demonstrated that proliferation of tumor cells was significantly inhibited in the group overexpressed with mutated $\beta$-catenin. Moreover, this inhibition was reversible when $\beta$-catenin was knocked-down in stably overexpressed clones. Taken together with the observation that cells in those groups harboring B-catenin accumulation exhibited neurite extension, we postulate that $\beta$-catenin accumulation may provide certain effects on neuronal differentiation. The hypothesis was supported by a recent study which demonstrated a role of $B$-catenin/Wnt signaling pathway in differentiation of neural stem cells (13).

Our growth study in stable transfected clones demonstrated obviously slower proliferation rate in cell clones with mutated B-catenin. This result differed from that of transient transfection model, in which growth suppression effect of del-E3 plasmid appeared milder than del-TCT45. The disparity was possibly the result of a minute difference in transfection efficiency or inequality in cellular reaction to each plasmid. The cell cycle study suggested that the growth retardation was a result of G1/S transition defect which could be shown in groups of cell clone transfected with mutated form of $\beta$-catenin. Based on cytometric evidence alone, although we cannot explain the negative growth effect of neuroblastoma cells by the cell-cycle exit and acquisition to the differentiation process, the pattern of cell-kinetics alteration was similar to that reported in trkA overexpressed cells in the presence of NGF (20). Taken together with our evidence of increased trkA expression in $\beta$-catenin overexpressed groups, it could be postulated that $\beta$-catenin indirectly enhanced differentiation in the NB-1 neuroblastoma cells, through an activation of NGF/trkA pathway.

Role of tyrosine kinase-A (trkA) in promoting morphologic differentiation and growth inhibition in neuroblastoma cell lines has been well defined (21). To promote neuronal 
differentiation, trkA functions in concert with its ligand NGF through autophosphorylation and downstream activation of several downstream genes including ras and mitogen-activated protein kinase 1. Increased expression of Ha-ras detected in our study confirmed the up-regulation of the signaling pathway and suggested a possible association between Wnt/Bcatenin and NGF/trkA cascades.

A previous study reported no significant effect of direct B-catenin overexpression in a neuroblastoma cell line N2A cell, in terms of morphological differentiation (22), for which the authors explained that the experiments were performed in serum-free condition. The findings support our postulation that B-catenin induced morphological differentiation through an increased expression of trkA, which functions in the presence of its ligand NGF. Further study in more detail is necessary to verify this potential connection.

In summary, our study demonstrated that accumulation of ß-catenin in a neuroblastoma cells led to growth inhibition and neurite extension which was related to up-regulation of trkA. The data suggest a possible availability of B-catenin as a molecular target for manipulation in the management of neuroblastoma.

\section{Acknowledgements}

This study was partially supported by a Grant-in-Aid from the Ministry of Education, Sciences, Sports and Culture of Japan (Grant no. 6591785). S.S. is supported by the Royal Thai Government via the Faculty of Medicine, Prince of Songkla University, Thailand.

\section{References}

1. Brodeur GM: Neuroblastoma: biological insights into a clinical enigma (Review). Nat Rev Cancer 3: 203-216, 2003.

2. Shimada H, Umehara S, Monobe Y, Hachitanda Y, Nakagawa A, Goto S, Gerbing RB, Stram DO, Lukens JN and Matthay KK: International neuroblastoma pathology classification for prognostic evaluation of patients with peripheral neuroblastic tumors: a report from the Children's Cancer Group. Cancer 92: 2451-2461, 2001.

3. Chatten J, Shimada H, Sather HN, Wong KY, Siegel SE and Hammond GD: Prognostic value of histopathology in advanced neuroblastoma: a report from the Childrens Cancer Study Group. Hum Pathol 19: 1187-1198, 1988.

4. Haas D, Ablin AR, Miller C, Zoger S and Matthay KK: Complete pathologic maturation and regression of stage IVS neuroblastoma without treatment. Cancer 62: 818-825, 1988.

5. Ambros PF, Ambros IM, Strehl S, Bauer S, Luegmayr A, Kovar H, Ladenstein R, Fink FM, Horcher E, Printz G, Mutz I, Schilling F, Urban C and Gadner H: Regression and progression in neuroblastoma. Does genetics predict tumour behaviour? Eur J Cancer 31A: 510-515, 1995.
6. Sidell N, Altman A, Haussler MR and Seeger RC: Effects of retinoic acid (RA) on the growth and phenotypic expression of several human neuroblastoma cell lines. Exp Cell Res 148: 21-30, 1983.

7. Garcia-Perez J, Avila J and Diaz-Nido J: Lithium induces morphological differentiation of mouse neuroblastoma cells. J Neurosci Res 57: 261-270, 1999.

8. Edsjo A, Nilsson H, Vandesompele J, Karlsson J, Pattyn F, Culp LA, Speleman F and Pahlman S: Neuroblastoma cells with overexpressed MYCN retain their capacity to undergo neuronal differentiation. Lab Invest 84: 406-417, 2004.

9. Maris JM and Mattthay KK: Molecular biology of neuroblastoma. J Clin Oncol 17: 2264-2279, 1999.

10. Bogenmann E, Peterson S, Maekawa K and Matsushima H: Regulation of NGF responsiveness in human neuroblastoma. Oncogene 17: 2367-2376, 1998.

11. Polakis P: Wnt signaling and cancer. Genes Dev 14: 1837-1851, 2000.

12. Satoh J and Kuroda Y: B-catenin expression in human neural cell lines following exposure to cytokines and growth factors. Neuropathology 20: 113-123, 2000.

13. Israsena N, Hu M, Fu W, Kan L and Kessler JA: The presence of FGF2 signaling determines whether $B$-catenin exerts effects on proliferation or neuronal differentiation of neural stem cells. Dev Biol 268: 220-231, 2004.

14. Nagasawa Y, Miyoshi Y, Iwao K, Shinomura Y, Matsuzawa Y and Nakamura Y: Transformation and morphological changes of murine $\mathrm{L}$ cells by transfection with a mutated form of betacatenin. Cancer Res 59: 3539-3542, 1999.

15. Sangkhathat S, Kusafuka T, Miao J, Yoneda A, Nara K, Yamamoto S, Kaneda Y and Fukuzawa M: In vitro RNA interference against beta-catenin inhibits the proliferation of pediatric hepatic tumors. Int J Oncol 28: 715-722, 2006.

16. Koch A, Denkhaus D, Albrecht S, Leuschner I, von Schweinitz D and Pietsch T: Childhood hepatoblastomas frequently carry a mutated degradation targeting box of the beta-catenin gene. Cancer Res 59: 269-273, 1999.

17. Udatsu Y, Kusafuka T, Kuroda S, Miao J and Okada A: High frequency of beta-catenin mutations in hepatoblastoma. Pediatr Surg Int 17: 508-512, 2001

18. Galderisi U, Jori FP and Giordano A: Cell cycle regulation and neuronal differentiation. Oncogene 22: 5298-5219, 2003

19. Ohira M, Kageyama H, Mihara M, Furuta S, Machida T, Shishikura T, Takayasu H, Islam A, Nakamura Y, Takahashi M, Tomioka N, Sakiyama S, Kaneko Y, Toyoda A, Hattori M, Sakaki Y, Ohki M, Horii A, Soeda E, Inazawa J, Seki N, Kuma H, Nozawa I and Nakagawara A: Identification and characterization of a 500-kb homozygously deleted region at 1p36.2-p36.3 in a neuroblastoma cell line. Oncogene 19: 4302-4307, 2000.

20. Kokunai T, Iguchi $\mathrm{H}$ and Tamaki N: Differentiation and growth inhibition of glioma cells induced by transfer of trkA protooncogene. J Neurooncol 42: 23-34, 1999.

21. Schramm A, Schulte JH, Astrahantseff K, Apostolov O, Limpt V, Sieverts H, Kuhfittig-Kulle S, Pfeiffer P, Versteeg R and Eggert A: Biological effects of TrkA and TrkB receptor signaling in neuroblastoma. Cancer Lett 228: 143-153, 2005.

22. Fan S, Ramirez SH, Garcia TM and Dewhurst S: Dishevelled promotes neurite outgrowth in neuronal differentiating neuroblastoma 2A cells, via a DIX-domain-dependent pathway. Brain Res Mol Brain Res 132: 38-50, 2004. 